\title{
Reduced Form Estimation Of The Noise Trading Model
}

S. Maheswaran, Institute for Financial Management and Research, India

G. Balasubramanian, Institute for Financial Management and Research, India C.A. Yoonus, Institute for Financial Management and Research, India

\begin{abstract}
In this paper, we develop and implement the reduced form version of the Noise Trading Model in the Indian stock market, which we had proposed in an earlier paper. We show how to estimate the model without making any assumptions regarding the cross-sectional dependence that may exist among the individual stocks. This allows us to come up with precise estimates of the share of information versus noise in the opening stock price. To be specific, information accounts for $52 \%$ of the variance of the opening stock price and noise contributes to the rest. When we split the overall sample into weekend versus weekday, we find that the information share of the opening price is significantly higher after a weekend at $72 \%$ compared to $46 \%$ during the rest of the week.
\end{abstract}

Keywords: Noise in Stock Prices; Information Share; Asset Pricing; Market Inefficiency; Market Microstructure

\section{INTRODUCTION}

e $\mathrm{n}$ an earlier paper, we had proposed the Noise Trading Model to explain the observed negative correlation between the overnight return and the day time return that we had documented among every single company constituting the NIFTY index, which plays a major role in the Indian stock market. Essentially, the Noise Trading Model is an unobserved components model in which the opening stock price contains a noise component that is assumed to be orthogonal to the change in the true (but unobserved) stock price caused by the arrival of information. We had argued that due to price discovery, the opening noise gets dissipated during trading, resulting in the closing prices being noise-free. (See Maheswaran et al. (2011) for more details.)

In this paper, we develop and implement the reduced form version of the Noise Trading Model. We show how to estimate the model without making any assumptions regarding the cross-sectional dependence that may exist among the individual stocks. This allows us to come up with precise estimates of the share of information versus noise in the opening stock price. To be specific, information accounts for $52 \%$ of the variance of the opening price change in the overall sample and noise contributes to the rest. When we split the overall sample into weekend versus weekday, what we find is that the information share is significantly higher after a weekend at $72 \%$, compared to $46 \%$ during the rest of the week.

Furthermore, we are able to break this down by showing that this is because the weekend-to-weekday variance ratio of the information variables is more than unity (meaning, higher after a weekend compared to a weekday) and that its counterpart in the opening noise is less than 1. Thus, both the effects move in the same direction. This evidence clearly establishes that there is more information after a weekend; i.e., from Friday close to Monday close, compared to a typical weekday, because the variance of the information components is higher and that of the opening noise is lower.

\section{LITERATURE REVIEW}

In finance and economics, most of the models developed are based on the assumption that the market is efficient and that traders are rational. Since by assumption traders are rational, the market becomes perfect whereby the theoretical model can be easily solved. However, in the past decade, several anomalies have been witnessed in 
the financial markets which cannot happen under the efficient market hypothesis, Schwert (2003). This had led recent finance literature to study the anomalies and seek to understand inefficient markets.

For instance, Figlewski (1978) studied market efficiency when there are heterogeneous traders, with the idea being that the degree of market efficiency should depend on the underlying characteristics of market participants. If there are more risk averse and homogeneous traders in the market, then we would expect the market to be more efficient. However, since the participants in the actual marketplace are heterogeneous, with a wide range of forecasting ability and diversity of expectation, the market can deviate relatively far from the benchmark of efficiency.

Black (1986) has discussed the importance of noise in financial markets. Noise makes trading in financial markets possible and thus allows us to observe prices. Noise may make the market inefficient, but it also prevents us from taking advantage of inefficiencies. Noise trading is essential for the existence of a liquid market. If there is no noise trading then there will be very little trading in the asset, because both traders (those who want buy or sell) will think that the other party has better information than him and hence will be reluctant to trade. Black's statement on noise and noise trading provides the essential missing ingredient. Noise trading is trading on noise as if it were information. People who trade on noise are willing to trade even though from an objective point of view would be better off not trading. Perhaps they think that the noise they are trading on is information, or they just like to trade. He argues that noise traders as a group lose money by trading while informed traders as a group will make money.

Kyle (1985) comes up with a theoretical framework where the model has three kinds of traders: a single risk neutral insider (who has unique access to private information of the ex post liquidation value of the risky asset), random noise traders (who trade randomly) and competitive risk neutral market makers (who set price efficiently conditional on the information they have about quantities traded by others). He considered that the insider (informed trader) makes a positive profit by exploiting his information, where the noise traders create a shadow for the informed trader from the market makers. In this model, there is noise in the opening price, but the information of the insider is gradually incorporated into prices through trading, thus leaving the closing price noise-free. This is the defining property of the Noise Trading Model that we make use of in this paper.

In another strand of literature, as in Shleifer and Vishny (1990), it is shown that the rational behavior of arbitrageurs leads to greater mispricing of long-term assets than short term assets. A short-term asset is one where mispricing must disappear in the near future, whereas mispricing of a long-term asset can persist for longer period of time because mispricing of claims of long-term investment projects takes longer to disappear, since it takes longer for fundamental uncertainty to be resolved. The risk arbitrage faces is noise trader risk, quite apart from fundamental risk. (see DeLong et al., 1990 also) For a long-term strategy there are several sources of risk: cost of fund for holding long-term, limit of fund (borrowing constraint), credit constraint (opportunity cost of money which is tied up long-term). Therefore, long-term trade is riskier than short term trade for the arbitrageur.

In DeLong et al. (1990), they introduced a simple model to understand noise trader risk in the asset market in which the noise trader has a random belief which affects the asset price and thereby creates more risk, wherein the asset price can diverge far away from fundamental value. Most of the time, economists ignore the presence of noise traders in the market (even when they exist in abundance) in discussions of price formation. According to Kyle (1985), irrational investors meet the rational arbitrageur who trades against them and in the process moves the price closer to fundamental value and as such, the impact of noise disappears from the closing prices.

In the Shleifer and Vishny (1990) paper, the authors study the limits of arbitrage, by examining the effectiveness of arbitrage in achieving market efficiency. Strictly speaking, the arbitrage which we see in the real market is risk arbitrage. In risk arbitrage, an arbitrageur does not make money with probability one, and may need substantial amount of capital to both execute his trade and cover his losses. Unlike the textbook definition of arbitrage, it is both risky and needs capital. (See Shleifer and Vishny, 1990.) But arbitrage plays a big role in the analysis of security market, because its effect is to bring the price to fundamental value and keep the market efficient. In this case, the implicit assumption is that, the market has a large number of small arbitrageurs and they take infinitesimal positions against mispricing. Since their position are too small and capital constraint is not binding, the arbitrageurs are risk neutral with respect to each trade. Their collective action drives the price to 
fundamental value. This kind of arbitrage is explained in the analysis of efficient market as in Fama (1965), and in models such as the CAPM as proposed by Sharpe (1964) and the APT in Ross (1976).

In Shiller (2003), the author launches an attack on the efficient market theory, using such well-documented findings as excess volatility and the feedback effect and also the fact that there is a substantial amount of noise that dominates the aggregate market movement. The efficient market theory is still not able to link market volatility and fundamentals. The idea of efficient market theory of the 1970's on speculative asset prices assumes that prices incorporate the best information available about fundamental values of the asset. Theoretical models of efficient markets have their place as characterization of ideal world; we cannot expect to see the pure form in action in actual financial markets.

French and Roll (1986) find that stock prices are much more volatile during exchange trading hours than during non-trading hours. They use data for all the companies which are traded in NYSE and AMEX for the period of 1963 to 1982 and calculated the return variances for weekday, weekend, holiday and holiday weekend for each two year sub-periods. They find that the weekend return from Friday close to Monday close has a variance that is on average $10.7 \%$ higher than on an ordinary trading day. One possible explanation for this kind of behavior is that information arrives more frequently during the business hours. Also they argue that if the trading noise is corrected quickly, the noise would increase intraday return variances. This is one of our motivations for defining a model, where it has been assumed that there is noise in the opening price but that it vanishes at the end of the trading day. They also estimated autocorrelations for lag 1 to 15, to test any significance for the noise trading hypothesis. Neither the public nor the private information hypothesis predict any serial correlation, while trading noise hypothesis predicts that daily return will be negatively auto correlated.

Previous empirical work analyzing the differential behavior of overnight return, compared to the day time return, includes Masulis and Ng (1995) and Wang et al (2009). The former paper studied the return dynamic properties of the overnight and day time return for the FTSE 100 index before and after the implementation of the electronic trading platform in the London Stock Exchange (LSE) and around the stock market crash of October 1987. The latter paper analyzed the different components of the close to close return (CCR), daytime return (DTR) and overnight (ONR), and their properties for NYSE listed stocks. In particular, they found that the correlation between overnight return and daytime return is insignificant.

In our previous paper (Maheswaran et al (2011)), we have proposed the Noise Trading Model in the framework of Kyle (1985) to explain the observed pattern of negative correlation between the overnight return and the day time return in the Indian stock market. We find that the opening stock price contains noise on an everyday basis but that the impact of noise does get eliminated from prices at the end of the trading day, which says something remarkable about the price discovery process inherent in the trading mechanism at work in India. We also show how to profit from this noise by coming up with a simple trading strategy and demonstrate that it is indeed highly profitable.

\section{MODEL SPECIFICATION}

Let $\mathrm{P}_{\mathrm{i}, \mathrm{t}}^{\mathrm{o}}$ be the opening price and $\mathrm{P}_{\mathrm{i}, \mathrm{t}}^{\mathrm{c}}$ the closing price on day $t$ for company $i$. Let's split the close-toclose return (CCR) into two parts, viz., the overnight return (ONR) and daytime return (DTR), defined as:

$$
\begin{aligned}
& \operatorname{CCR}_{\mathrm{i}, \mathrm{t}}=\log \frac{\mathrm{P}_{\mathrm{i}, \mathrm{t}}^{\mathrm{c}}}{\mathrm{P}_{\mathrm{i}, \mathrm{t}-1}^{\mathrm{c}}} \\
& \mathrm{DTR}_{\mathrm{i}, \mathrm{t}}=\log \frac{\mathrm{P}_{\mathrm{i}, \mathrm{t}}^{\mathrm{c}}}{\mathrm{P}_{\mathrm{i}, \mathrm{t}}^{\mathrm{o}}}
\end{aligned}
$$


$\mathrm{ONR}_{\mathrm{i}, \mathrm{t}}=\log \frac{\mathrm{P}_{\mathrm{i}, \mathrm{t}}^{\mathrm{o}}}{\mathrm{P}_{\mathrm{i}, \mathrm{t}-\mathrm{l}}^{\mathrm{c}}}$

where $\mathrm{CCR}_{\mathrm{i}, \mathrm{t}}, \mathrm{DTR}_{\mathrm{i}, \mathrm{t}}$ and $\mathrm{ONR}_{\mathrm{i}, \mathrm{t}}$ are the close-to-close return (CCR), daytime return (DTR) and overnight return (ONR) respectively for each company $i$ on day $t$.

In this paper, we are interested in studying the cross-sectional properties and so, we shall drop the time subscript $t$ in the notation. We assume that the news related to both the market and the individual firm that are released during the trading day are incorporated in the closing price, in keeping with Kyle (1985). The information that comes after the trading hours will be evaluated by the traders with some noise, because there is no way for them to infer one another's private value, given that the market is closed. As a result, there will be some opening noise early in the trading day, which will get dissipated by the end of the trading day due to price discovery.

Let's denote the overnight true price change of company $i$ to be $\eta_{\mathrm{i}}$, the true price change during the trading day of company $i$ to be $\Delta X_{\mathrm{i}}$ and the opening noise by $\mathrm{Y}_{\mathrm{i}}(0)$. Let's define the model by relating the definition of returns given in the above three equations in terms of information and noise terms as follows:

$$
\begin{aligned}
& \mathrm{CCR}_{\mathrm{i}}=\eta_{\mathrm{i}}+\Delta \mathrm{X}_{\mathrm{i}} \\
& \mathrm{DTR}_{\mathrm{i}}=\Delta \mathrm{X}_{\mathrm{i}}-\mathrm{Y}_{\mathrm{i}}(0) \\
& \mathrm{ONR}_{\mathrm{i}}=\eta_{\mathrm{i}}+\mathrm{Y}_{\mathrm{i}}(0)
\end{aligned}
$$

\section{Reduced Form}

We will assume henceforth that $\eta_{i}, \Delta X_{i}$ and $Y_{i}(0)$ are mutually orthogonal to each other for each fixed company $i$. We will also assume that they are identically distributed across company $i$, but we will refrain from imposing any specific structure on the cross-sectional dependence across $i$, because we would like to explore the reduced form version of the model.

Our goal in proposing this reduced form version of the Noise Trading Model is to estimate the average variance of each of the components in the returns and, in particular, to measure the share of the information in the variance of the opening price for the overall sample as well as the weekend and weekday sub-samples. To enable us to do that, we start by deriving the relevant equations to estimate the average variance of each component:

$$
\begin{aligned}
\operatorname{Avg}(\operatorname{Var}(\mathrm{ONR})) & =\operatorname{Var}\left(\mathrm{ONR}_{\mathrm{i}}\right) \\
& =\operatorname{Var}\left(\eta_{\mathrm{i}}+\mathrm{Y}_{\mathrm{i}}(0)\right) \\
& =\sigma_{\eta}^{2}+\sigma_{\mathrm{Y}(0)}^{2}
\end{aligned}
$$

$$
\begin{aligned}
\operatorname{Avg}(\operatorname{Var}(\mathrm{DTR})) & =\operatorname{Var}\left(\mathrm{DTR}_{\mathrm{i}}\right) \\
& =\sigma_{\Delta \mathrm{X}}^{2}+\sigma_{\mathrm{Y}(0)}^{2}
\end{aligned}
$$

$$
\begin{aligned}
\operatorname{Avg}(\operatorname{Var}(\mathrm{CCR})) & =\operatorname{Var}\left(\mathrm{CCR}_{\mathrm{i}}\right) \\
& =\sigma_{\eta}^{2}+\sigma_{\Delta \mathrm{X}}^{2}
\end{aligned}
$$


Table 1: Summary of the Data

\begin{tabular}{|l|c|c|c|}
\hline & Overall & Weekend & Weekday \\
\hline ONR & \multicolumn{3}{|c|}{$\mathbf{A v g ( V a r ( ) ) ^ { * 1 0 } \mathbf { - 3 } ^ { - 3 }}$} \\
\hline DTR & 0.212 & 0.266 & 0.230 \\
\hline CCR & 0.841 & 0.930 & 0.947 \\
\hline
\end{tabular}

Source: Prowess

At the same time, as reported in Table 1, we have the following estimates from the data, for the overall sample, for each of the left-hand side quantities above:

$$
\operatorname{Avg}\left(\operatorname{Var}(\mathrm{ONR})=0.212 * 10^{-3}\right.
$$

$$
\operatorname{Avg}\left(\operatorname{Var}(\mathrm{DTR})=0.841 * 10^{-3}\right.
$$

$$
\operatorname{Avg}\left(\operatorname{Var}(C C R)=0.851 * 10^{-3}\right.
$$

Combining the two sets of equations, we get that

$$
\begin{gathered}
\sigma_{\eta}^{2}+\sigma_{\mathrm{Y}(0)}^{2}=0.212 * 10^{-3} \\
\sigma_{\Delta \mathrm{X}}^{2}+\sigma_{\mathrm{Y}(0)}^{2}=0.841 * 10^{-3} \\
\sigma_{\eta}^{2}+\sigma_{\Delta \mathrm{X}}^{2}=0.851 * 10^{-3}
\end{gathered}
$$

Table 2: Reduced Form Estimates

\begin{tabular}{|l|c|c|c|c|}
\hline & Overall & Weekend & Weekday & Weekend to weekday variance ratio \\
\hline $\boldsymbol{\sigma}_{\boldsymbol{\Lambda x}}^{2}$ & $7.40 \mathrm{E}-04$ & $8.56 \mathrm{E}-04$ & $7.12 \mathrm{E}-04$ & 1.20 \\
\hline $\boldsymbol{\sigma}_{\boldsymbol{\eta}}^{\mathbf{2}}$ & $1.11 \mathrm{E}-04$ & $1.92 \mathrm{E}-04$ & $9.07 \mathrm{E}-05$ & 2.12 \\
\hline $\boldsymbol{\sigma}_{\mathbf{Y}(\mathbf{0})}^{\mathbf{2}}$ & $1.01 \mathrm{E}-04$ & $7.39 \mathrm{E}-05$ & $1.08 \mathrm{E}-04$ & 0.69 \\
\hline $\boldsymbol{\beta}$ & 0.523 & 0.722 & 0.457 & \\
\hline
\end{tabular}

Thus, we have three simultaneous equations in three unknowns, which can be easily solved, the results of which are given in Table 2. For instance, for the overall sample, the estimates of $\sigma_{\Delta \mathrm{X}}^{2}, \sigma_{\eta}^{2}$ and $\sigma_{\mathrm{Y}(0)}^{2}$ are given by $0.740 * 10^{-3}, 0.111 * 10^{-3}$ and $0.101 * 10^{-3}$, respectively.

It has been shown in our previous paper, Maheswaran et al (2011), that the information share of the opening stock price is given by $\beta$, where $\beta$ is the slope coefficient from a regression of the close-to-close return $(\mathrm{CCR})$ on the overnight return (ONR). Notice from Equations (1) and (3) that $\operatorname{Cov}(\mathrm{ONR}, \mathrm{CCR})=\operatorname{Var}(\eta)$ and $\operatorname{Var}(\mathrm{ONR})=\operatorname{Var}(\eta)+\operatorname{Var}(\mathrm{Y}(0))$ and so,

$$
\begin{aligned}
\beta & =\frac{\operatorname{Cov}(\mathrm{ONR}, \mathrm{CCR})}{\operatorname{Var}(\mathrm{ONR})} \\
& =\frac{\operatorname{Var}(\eta)}{\operatorname{Var}(\eta)+\operatorname{Var}(\mathrm{Y}(0))}
\end{aligned}
$$


Now that we have the estimates of the variance of the components in hand, we can plug them into Equation (13) to obtain the information share of the opening price. As reported in Table 2, the information share turns out to be $52.3 \%$ in the overall sample.

\section{DATA DESCRIPTION}

The NIFTY Index and its constituent companies from January 2001 to December 2010 are used in this study. The NIFTY Index consists of well diversified 50 liquid stocks from 22 sectors. The Prowess database provided by CMIE (Centre for Monitoring the Indian Economy Pvt. Ltd.) was used to extract the adjusted opening and closing stock price series.

\section{EMPIRICAL FINDINGS}

We split the close-to-close return (CCR) into overnight return (ONR) and daytime return (DTR) for all weekdays using the formula given in the model section. The ONR for the weekend is defined as the log difference between Friday close and Monday open, and similarly DTR for the weekend is defined as Monday open to Monday close, with the close-to-close return for the weekend is defined as Friday close to Monday close. We will assume that the price on a Monday reflects the information over the weekend. From Table 1, we can see that the average variance of DTR is higher than that of ONR, by almost four times, something which is in agreement with the finding of French and Roll (1986) that equity returns more volatile during trading hours than non-trading hours. Also notice that the average variance of DTR after a weekend is $0.930 * 10^{-3}$, which is higher than that of other weekdays. Most of the variance in CCR is contributed by the daytime return (DTR), which is in agreement with Wang et al (2009) where the same feature was found among NYSE listed stocks.

We can see from Table 2 that $\beta$, the measure of information share in the opening stock price, is 0.523 in the overall sample, which is similar to what we had computed as the cross-sectional average of all the $\beta$ 's of the individual companies in our previous paper, Maheswaran et al. (2001). Notice also from Table 2 that the weekend to weekday ratio for the variance of information components is more than 1 and it is less than 1 for the opening noise component. That is to say,

$$
\begin{aligned}
\text { Weekend } \sigma_{\eta}^{2} / \text { Weekday } \sigma_{\eta}^{2} & =2.12 \\
\text { Weekend } \sigma_{\Delta \mathrm{X}}^{2} / \text { Weekday } \sigma_{\Delta \mathrm{X}}^{2} & =1.20 \\
\text { Weekend } \sigma_{\mathrm{Y}(0)}^{2} / \text { Weekday } \sigma_{\mathrm{Y}(0)}^{2} & =0.69
\end{aligned}
$$

What we do next is to assess the evidence in the data on the significance of the differences between the estimates of the variance components over the weekend versus a typical weekday. To do this, we undertake a bootstrap experiment as follows:

1. Choose a random sample with replacement of the same length as in the original sample.

2. Estimate the variance of the unobservable components as given in Equations 10, 11 and 12

3. Repeat this procedure to create a thousand such replications.

4. Then, compute the mean and standard deviation of the 1,000 such bootstrap estimates.

Table 3: Test for Differences between Weekend and Weekday

\begin{tabular}{|l|c|c|c|c|}
\hline & $\boldsymbol{\sigma}_{\Delta \mathbf{x}}^{2}$ & $\boldsymbol{\sigma}_{\boldsymbol{\eta}}^{2}$ & $\boldsymbol{\sigma}_{\mathbf{Y}(\mathbf{0})}$ & $\boldsymbol{\beta}$ \\
\hline Actual(Diff) & $1.45 \mathrm{E}-04$ & $1.01 \mathrm{E}-04$ & $-3.38 \mathrm{E}-05$ & 0.27 \\
\hline Std Error & $2.17 \mathrm{E}-05$ & $9.14 \mathrm{E}-06$ & $1.10 \mathrm{E}-05$ & 0.04 \\
\hline t-stat & 6.68 & 11.09 & -3.07 & 7.39 \\
\hline
\end{tabular}


In particular, we are interested in testing whether the differences are significant when we consider a weekend versus weekday split, in terms of the estimates of the variance of the unobservable components. In Table 3 , we report the standard error using the bootstrap procedure outlined above, from which we can see that all the tstatistics are large (in absolute value): 11.09 for $\sigma_{\eta}^{2}$ and 6.68 for $\sigma_{\Delta \mathrm{X}}^{2}$ (which are the variances of the information components) and -3.07 for $\sigma_{\mathrm{Y}(0)}^{2}$, which is the variance of the opening noise. This evidence clearly establishes that there is more information after a weekend compared to a typical weekday, because the variances of the information components are higher and that of the opening noise is lower.

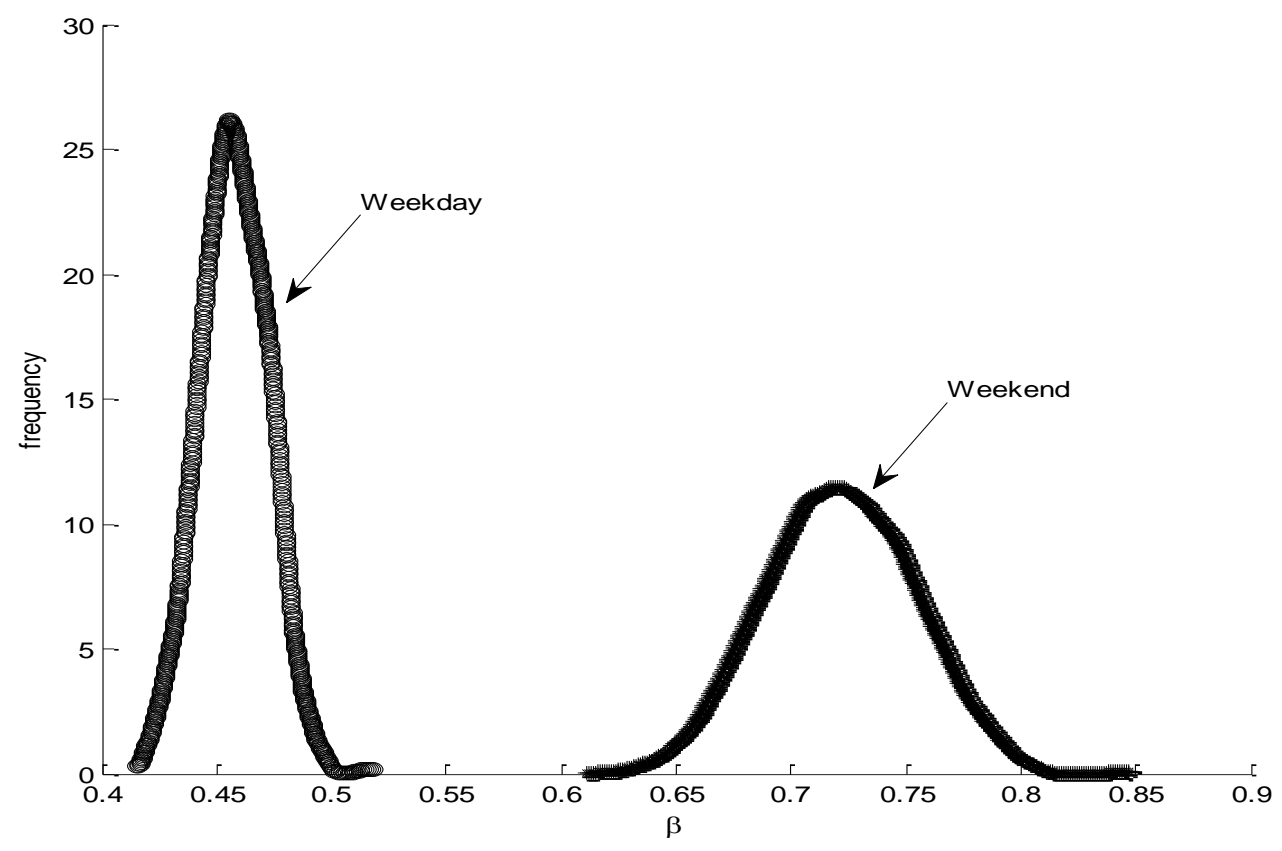

Figure 1: Information Share - Comparison between Weekend and Weekday

Furthermore, $\beta$ the measure of the informativeness of the opening price, is 0.722 after a weekend and 0.457 after a typical weekday, with a t-statistic of 7.39 for the difference between them, which clearly shows that there is more information in the opening price after a weekend than after a typical weekday, presumably because more information has accumulated over the course of the weekend when the market was closed, which gets revealed in the opening price on a Monday. This can also be seen in Figure 1 where we have plotted the empirical density of $\beta$, the information share of the opening price, over the weekend versus a typical weekday. It is clear that the empirical density of $\beta$ for the weekend is shifted toward the right of weekday, just as suggested by the t-test for the difference between them.

\section{CONCLUSION}

In this paper, we have developed and implemented the reduced form version of the Noise Trading Model in the Indian stock market, which we had proposed in an earlier paper. We have shown how to estimate the model without making any assumptions regarding the cross-sectional dependence that may exist among the individual stocks. This allows us to come up with precise estimates of the share of information versus noise in the opening prices. To be specific, information accounts for $52 \%$ of the variance of the opening price change in the overall sample. When we split the sample into weekend versus weekday, what we find is that the information share is significantly higher after a weekend at $72 \%$, compared to a typical weekday when it is around $46 \%$. Furthermore, 
we are able to break this down by showing that this is because the weekend-to-weekday variance ratio of the information variables is more than unity (meaning, higher after a weekend compared to a weekday) and that its counterpart in the opening noise is less than 1 . Thus, both the effects move in the same direction.

\section{AUTHOR INFORMATION}

S. Maheswaran is Professor with the Centre for Advanced Financial Studies (CAFS) at the Institute for Financial Management and Research (IFMR) in Chennai, India. He holds a Ph.D. from the University of Minnesota after having completed PGDM from IIM Calcutta and a B.Com. from Vivekananda College. He has taught at Washington University in St. Louis, Columbia University and the University of Wisconsin in Madison. E-mail: mahesh@ifmr.ac.in. Corresponding author

G. Balasubramanian has had two decades of experience in executive training, teaching, consulting and research in Accounting, Finance and Information Technology. He has been a visiting faculty for several African countries in the area of finance and information technology, under the sponsorship of the commonwealth secretariat. He has designed and implemented Decision Support Systems for major organizations. He has published papers in the area of capital markets, accounting, information technology and knowledge management. He has participated as a resource person in a study of disinvestments of public sector enterprises sponsored by the United States Agency for International Development (USAID). E-mail: bala@ifmr.ac.in

C. A. Yoonus is a Research Scholar at the Institute for Financial Management and Research in Chennai, India. Prior to joining the Ph.D. Program, he completed his Masters in Mathematics from Cochin University of Science and Technology (CUSAT) and a PGDM in Finance from IFMR. His research interests are in Asset Pricing, Market Microstructure and Financial Economics. E-mail: kalanad@gmail.com

\section{REFERENCES}

1. $\quad$ Black, F. (1986). Noise, Journal of Finance, 41 (3), 529-543.

2. DeLong, J. B., A. Shleifer, L. H. Summers, and R. J. Waldmann (1990). Noise trader risk in financial markets, Journal of Political Economy, 98 (4), 703-738.

3. Fama, E. F. (1965). The behavior of stock-market prices, Journal of Business, 38 (1), 34-105.

4. Figlewski, S. C. (1978). Market efficiency in a market with heterogeneous information, Journal of Political Economy, 86 (4), 581-97.

5. French, K R. \& Roll, R. (1986). Stock return variances: The arrival of information and the reaction of traders, Journal of Financial Economics, 17 (1), 5-26.

6. Kyle, A. S. (1985). Continuous auctions and insider trading, Econometrica, 53 (6), 1315-35.

7. Maheswaran, S., Balasubramanian, G. \& Yoonus, C. A. (2011). Opening jump and noise trading, SSRN eLibrary

8. Masulis, R. W. and V. Ng (1995). Overnight and daytime stock return dynamics on the London Stock Exchange, Journal of Business and Economic Statistics, 13 (4), 365-378.

9. $\quad$ Ross, S. A. (1976). The arbitrage theory of capital asset pricing, Journal of Economic Theory, 13 (3), 341360.

10. Schwert, G. W. (2003). Anomalies and market efficiency. In G. Constantinides, M. Harris, and R. M. Stulz (Eds.), Handbook of the Economics of Finance, Volume 1 of Handbook of the Economics of Finance, Chapter 15, (pp. 939-974). Elsevier.

11. Sharpe, W. F. (1964). Capital asset prices: A theory of market equilibrium under conditions of risk, The Journal of Finance, 19 (3), 425-442.

12. Shiller, R. J. (2003). From efficient markets theory to behavioral finance, The Journal of Economic Perspectives, 17 (1), 83-104.

13. Shleifer, A. and R. W. Vishny (1990). Equilibrium short horizons of investors and firms, American Economic Review, 80 (2), 148-53.

14. Shleifer, A. and R. W. Vishny (1997). The limits of arbitrage, The Journal of Finance, 52 (1), 35-55.

15. Wang, F., S.-J. Shieh, S. Havlin, and H. E. Stanley (2009). Statistical analysis of the overnight and daytime return, Physical Review, 79 (5), 056109. 